\title{
Video Article \\ Investigating the Effects of Antipsychotics and Schizotypy on the N400 Using Event-Related Potentials and Semantic Categorization
}

\author{
Vivian $\mathrm{Gu}^{1}$, Ola Mohamed $\mathrm{Ali}^{1}$, Katherine L'Abbée Lacas ${ }^{2}$, J. Bruno Debruille ${ }^{3,4}$ \\ ${ }^{1}$ Department of Psychology, McGill University \\ ${ }^{2}$ Department of Cognitive Science, McGill University \\ ${ }^{3}$ Douglas Institute, Department of Psychiatry, McGill University \\ ${ }^{4}$ Neurology and Neurosurgery Department, McGill University
}

Correspondence to: J. Bruno Debruille at j.bruno.debruille@gmail.com

URL: https://www.jove.com/video/52082

DOI: doi:10.3791/52082

Keywords: Behavior, Issue 93, Electrical brain activity, Semantic categorization, Event-related brain potentials, Neuroscience, Cognition, Psychiatry, Antipsychotic medication, N400, Schizotypy, Schizophrenia.

Date Published: $11 / 19 / 2014$

Citation: Gu, V., Mohamed Ali, O., L'Abbée Lacas, K., Debruille, J.B. Investigating the Effects of Antipsychotics and Schizotypy on the N400 Using Event-Related Potentials and Semantic Categorization. J. Vis. Exp. (93), e52082, doi:10.3791/52082 (2014).

\section{Abstract}

Within the field of cognitive neuroscience, functional magnetic resonance imaging (fMRI) is a popular method of visualizing brain function. This is in part because of its excellent spatial resolution, which allows researchers to identify brain areas associated with specific cognitive processes. However, in the quest to localize brain functions, it is relevant to note that many cognitive, sensory, and motor processes have temporal distinctions that are imperative to capture, an aspect that is left unfulfilled by fMRl's suboptimal temporal resolution. To better understand cognitive processes, it is thus advantageous to utilize event-related potential (ERP) recording as a method of gathering information about the brain. Some of its advantages include its fantastic temporal resolution, which gives researchers the ability to follow the activity of the brain down to the millisecond. It also directly indexes both excitatory and inhibitory post-synaptic potentials by which most brain computations are performed. This sits in contrast to fMRI, which captures an index of metabolic activity. Further, the non-invasive ERP method does not require a contrast condition: raw ERPs can be examined for just one experimental condition, a distinction from fMRI where control conditions must be subtracted from the experimental condition, leading to uncertainty in associating observations with experimental or contrast conditions. While it is limited by its poor spatial and subcortical activity resolution, ERP recordings' utility, relative cost-effectiveness, and associated advantages offer strong rationale for its use in cognitive neuroscience to track rapid temporal changes in neural activity. In an effort to foster increase in its use as a research imaging method, and to ensure proper and accurate data collection, the present article will outline - in the framework of a paradigm using semantic categorization to examine the effects of antipsychotics and schizotypy on the N400 - the procedure and key aspects associated with ERP data acquisition.

\section{Video Link}

The video component of this article can be found at https://www.jove.com/video/52082/

\section{Introduction}

While neuroimaging advances have undeniably augmented our understanding of the functions of the brain, these advances have a largely static emphasis on elucidating the structures of the brain. This subsequently leaves the dynamic temporal features of brain networks relatively shrouded in obscurity, as neuroimaging methods that do address these temporal aspects, such as fMRI and near-infrared spectroscopy (NIRS), produce temporal resolutions that are inadequate compared to that of EEG. It is thus pertinent to better understand and employ the use of ERP recording, which is able to produce vast amounts of meaningful information that otherwise would be unknown. ERP recording is a noninvasive analytical tool that allows cognitive neuroscientists to track the rapid temporal modulations of neural activity that are elicited by cognitive sensory, and motor processes. It involves placing electrodes on the scalp to capture the electrical postsynaptic responses of large neuronal populations ${ }^{2}$ to specific events or stimuli. ERPs are electroencephalographic changes that are time-locked to sensory, motor, or cognitive events, and are believed to represent the summation of postsynaptic potentials produced during information processing ${ }^{3}$. These ERP waveforms are characterized by their latency, amplitude, polarity, and scalp distribution, providing a rich array of neurological data that may stimulate further insight into the neural connections that contribute to cognition.

The goal of ERP recording and electroencephalography (EEG) is to obtain information about the foundational neural processes involved in higher-order, complex cognitive operations ${ }^{4}$. ERPs are defined by their negative or positive fluctuation, as well as their location on the scalp and the time in which they appear after the onset of the stimulus. Various ERPs have been associated with different aspects of cognitive processing. For example, we have been looking at the N400 event-related brain potential ${ }^{5}$, a negative waveform that is elicited $400 \mathrm{~ms}^{2}$ after the onset of meaningful stimuli such as words ${ }^{6}$, and whose distribution on the scalp is known to depend on the semantic category of these stimuli. The N400 is an index of semantic activations, and studies have shown that it has a larger amplitude for words which activate more representations, such 
as concrete words (e.g., banana) that activate both visual and verbal representations, than for abstract words (e.g., idea), which activate only verbal representations ${ }^{7,8}$. Research also supports the idea that even mild symptoms of schizophrenia, as measured by schizotypy scales, are associated with abnormally excessive semantic activation of the $\mathrm{N} 00^{9}$. As such, we are exploring whether antipsychotic medication, which decreases schizophrenic symptom presentation, normalizes abnormal semantic activations of the N400 in healthy individuals with high levels of schizotypy. Here, the use of ERPs in a semantic categorization paradigm is advantageous for studying the effects of antipsychotics on the N400.

As ERP recording is a non-invasive and relatively economical method to assess neural functioning, it can be applied in a wide range of domains, as evidenced by the substantial number of ERP studies in cognitive neuroscience, neurology, neuropsychology, psycholinguistics, and cognitive psychology. Its versatility enables investigators to ask pertinent research questions about the relative timing of neural events in a large variety of domains, including language, cognition, and the study of various psychiatric disorders ${ }^{10}$ such as schizophrenia, bipolar affective disorder, depression, and alcohol dependence disorder, and it offers substantial advantages over alternative methods of neuroimaging, such as functional magnetic resonance imaging (fMRI). Some of these include its excellent temporal resolution, which elucidates brain activity down to the millisecond. ERP recording also directly indexes excitatory post-synaptic potentials (EPSPs) and inhibitory post-synaptic potentials (IPSPs) through which many brain computations are performed, something that fMRI does not do. Another advantage of ERP recording is that it offers the possibility of distinguishing inhibitory activity (neuronal hyperpolarizations) from excitatory activity (neuronal depolarization), whereas in fMRI, greater signals are not clearly differentiated between these two. Further, the ERP method does not require a contrast condition: raw ERPs can be examined for just one experimental condition, a distinction from fMRI where control conditions must be subtracted from the experimental condition, leading to uncertainty in associating observations with experimental or contrast conditions. ERP recordings' utility, relative costeffectiveness and associated advantages offer strong rationale for the use of ERPs in cognitive neuroscience as a way to track rapid temporal changes in neural activity. Followed is a step-by-step guide to the basics of running an EEG experiment and recording event-related potentials.

\section{Protocol}

This protocol follows the guidelines set forth by the Douglas Institute Research and Ethics Board.

\section{Patient Preparation}

1. When the participant arrives, explain the experiment and obtain a signature on an informed consent form that has received ethics approval from the institutions' Institutional Review Board.

2. Make a mark using the red pencil at the bridge of the nose (nasion), in between the eyebrows. Using a tape measure, measure from the nasion to the lower base of the skull, the inion. Discern this location by sliding a hand up the neck until a bump on the back of the participant's head is felt.

3. Take $1 / 10^{\text {th }}$ of the measurement between the nasion and the inion and make a mark up near the hairline from the nasion, taking care to ensure that these measurements are in line with the nose.

4. Wrap the Head Measuring Tape (4 Color ribbon) around the head at the level of the mark made in Step 1.3. Determine cap size based on where the non-colored end of the ribbon meets the colored end: use a blue cap if it crosses in the blue region, use a blue-red cap if it crosses between the blue and red regions, etc.

\section{Electrode Placement}

1. Place the cap onto the participant's head. Line up the two electrodes at the front of the cap with the eyes. Insert the disposable sponge disks on the frontal electrodes (FP1 and FP2), to keep the electro-gel from spreading.

2. Hold the two frontal electrodes against the mark near the hairline and pull the cap over the head. Ensure that the cap fits snugly on the participants head.

3. Place the ear electrodes onto the participant's earlobes as reference electrodes. Clean the earlobes with alcohol. Using a cotton tip applicator, spread EEG abrasive skin prepping gel onto the earlobes to facilitate conductivity.

4. Take a syringe and fill it with electrolyte gel. Then, squeeze the gel onto the ear electrode. Carefully place the gel-filled ear electrode onto the participant's earlobes.

5. Attach the wires from the cap and the ear electrodes to the EEG amplifier. Set the gain of the amplifier to 20,000. Attach the harness to the cap with the cap straps, making sure that it is snugly keeping the cap in place.

6. Start applying electrolyte gel to the ground electrode, which is found in the middle anterior of the cap. Electrolyte gel increases the electrical connectivity between the scalp and the electrode.

7. Use the syringe to squeeze electrolyte gel into the electrode. Start by inserting the syringe into the electrode and rock it back and forth until it is in contact with the scalp. Then, simultaneously squeeze the syringe and pull up, creating a vertical column of gel.

8. Repeat Step 2.7 for all of the electrodes.

9. Abrade the scalp underneath the electrodes with a blunt needle. Do this while observing the computer monitor, which should display a schematic of the electrodes. Aim to scratch the scalp until the corresponding electrode on the screen turns black in color. It is recommended to start with the ear electrodes, and then move on to the ground electrodes.

\section{Electroencephalography EEG Experiment}

1. Read the instructions for the experiment to the subject. Open the EEG acquisition software on the computer

2. Observe the resting activity of the electrodes. Troubleshoot for "bad" electrodes, such as flat line signals, or excessively active signals. If there are any, repeat Steps 2.7 and 2.9 to minimize impedance.

strong>NOTE: The impedance, which is measured using a $30 \mathrm{~Hz}$ current, should be kept below $5 \mathrm{k} \Omega$. 
3. Begin recording once the electrode signals look good and there are no flat line signals or excessively active signals. Look for any myograms or eye movement, and ensure that the participant refrains from blinking and tensing their jaw and forehead muscles, as these will produce excessive noise in the data. At rest, there may be beta and alpha rhythms present.

4. Start the experiment on the second computer. The timing of the experimental design is important. Observe carefully to ensure that the experiment software, such as E-Prime, sends "markers" to indicate stimulus onset. This is crucial as stimulus onset is often used as a reference point for an event in ERP paradigms.

5. Once the experiment is complete, ensure that the data has been saved- this process will differ depending on the software used. Carefully remove the cap, and assist the participant with hair washing and drying.

6. Detach the cap and ear electrodes from the amplifier, remove the disposable sponge disks, and clean the cap and ear electrodes under running water using a stick or the end of a cotton swab. Use a mild soap or shampoo, as well as a toothbrush, to gently clear the gel from the electrodes. Rinse thoroughly. Allow the equipment to air dry.

\section{Data Processing}

1. To obtain ERP components from the EEG, determine which epoch will be studied. For example, to compute the N400 amplitude, use the mean voltages in the $300-500$ msec time-window for calculation.

2. Before averaging the EEG epochs, reject trials with excessive eye movements or amplifier saturations. Depending on the experiment paradigm, it may be relevant to reject trials that correspond to incorrect responses.

3. After averaging, filter the ERPs and plot the data (see Animated Figure 1 for specific instructions on data processing using Matlab, EEGLab, and ERPLab).

\section{Representative Results}

Five figures have been included to aid researchers in obtaining optimal ERP recording. Figure 1 depicts ERP waveforms obtained following successful completion of this protocol; it constitutes a "good" result due to its accurate recording. Figure 2 on the other hand, illustrates ERP waveforms that would characterize a poor execution of the protocol, resulting in "bad" results, which are most notably identified in excessively noisy signals. Figure 3 shows the common problems that may arise in ERP recording that can impede proper data collection. High activity can be interpreted in the high frequency and large amplitudes in the waves. In contrast, a "flatline" indicates a lack of activity or improper connectivity. Figures $\mathbf{4}$ and $\mathbf{5}$ show the grand averages for the main results of our investigation into the effects of antipsychotics and schizotypy on the N400, as measured using a semantic categorization paradigm.

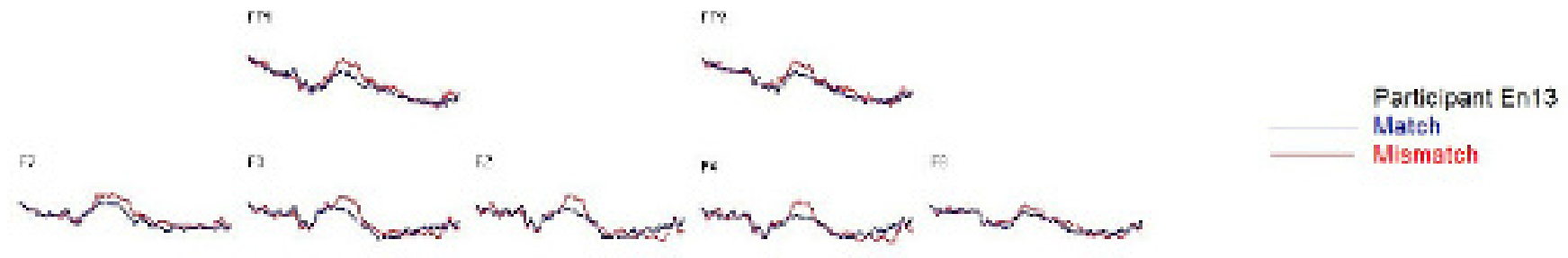

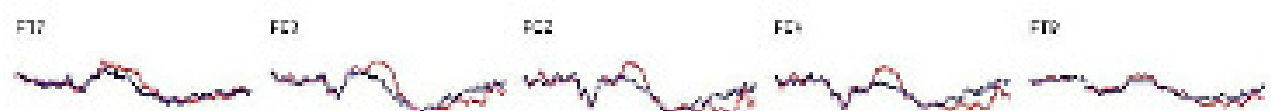

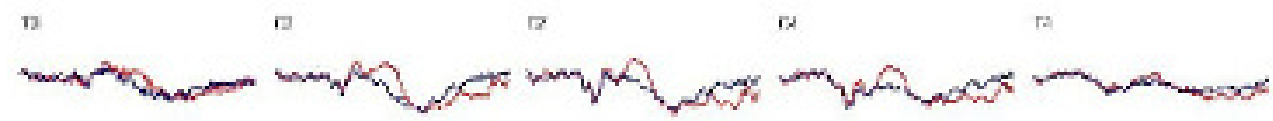

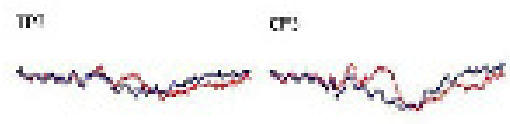

crs ins

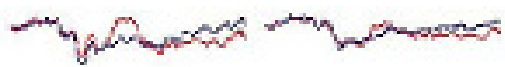

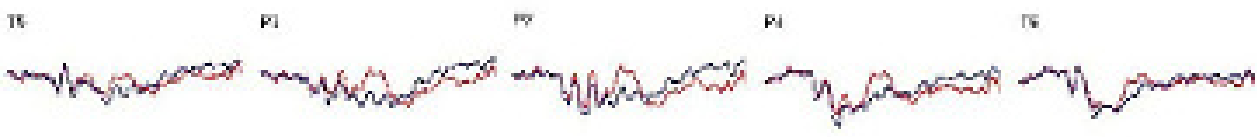

a

re<smiles>CCNC(=O)OCC</smiles>

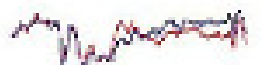

Figure 1. Good Result. This figure depicts the averaged ERP waveforms of one subject over all trials following successful execution of the technique, resulting in no flat lines or excessive noise. Note that the waves will look slightly different depending on the experimental paradigm used- tasks that rely heavily on different processes (cognitive, sensory, and motor) will produce different waveforms depending on the content of the task and the skills required by the task. Please click here to view a larger version of this figure. 


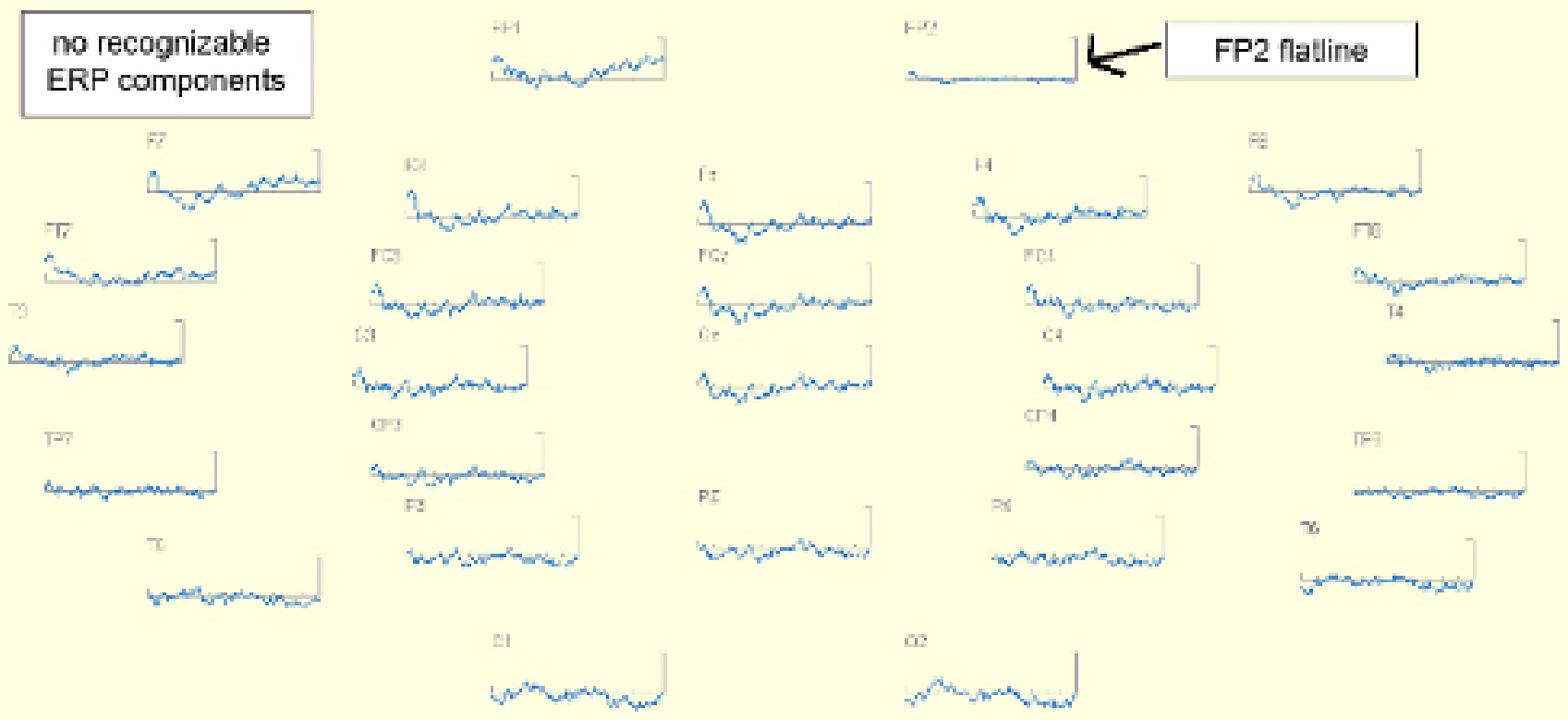

Figure 2. Poor Result. This figure depicts the averaged ERP waveforms of one subject over all trials following inadequate execution of the technique. The waveforms shown here are poor due to the noise, as well as the flat line in FP2. Note also the lack of recognizable ERP components in the EEG. Please click here to view a larger version of this figure.

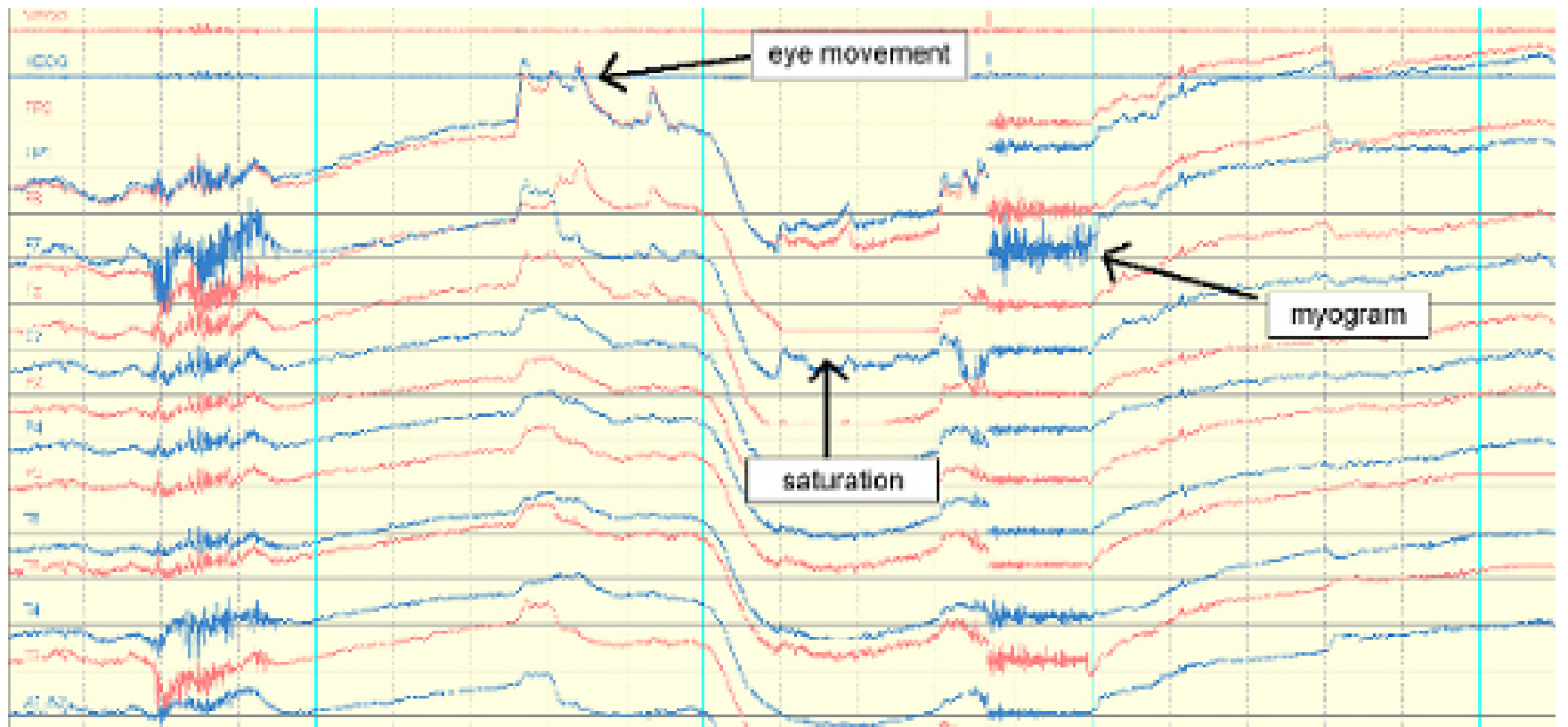

Figure 3. Common Problems. This figure depicts the common problems that arise with this technique; namely, eye movement, muscle movement, or saturation. Note that these problems can occur in any of the channels, so be sure to check all of them. Please click here to view a larger version of this figure. 

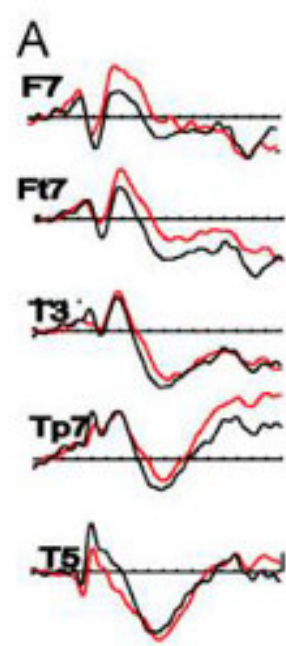

N1 peaks
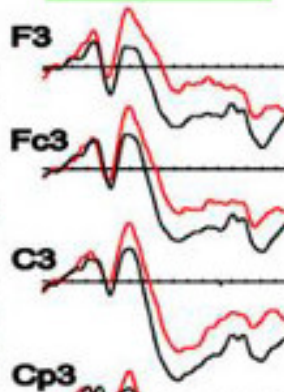

01 क्य

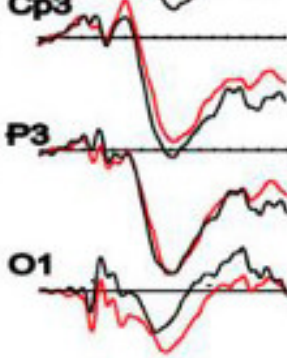

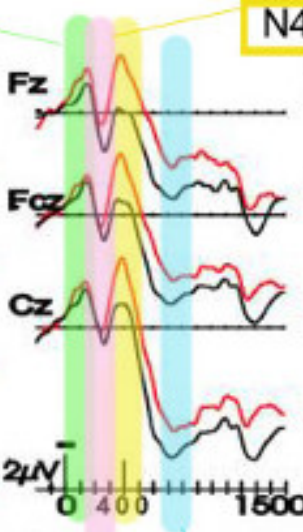

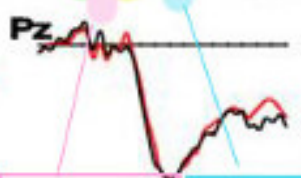

P2 peaks $\mathrm{P} 6$ peaks
N400 peaks

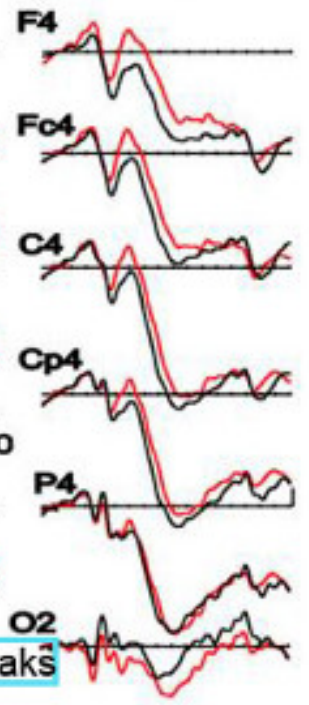

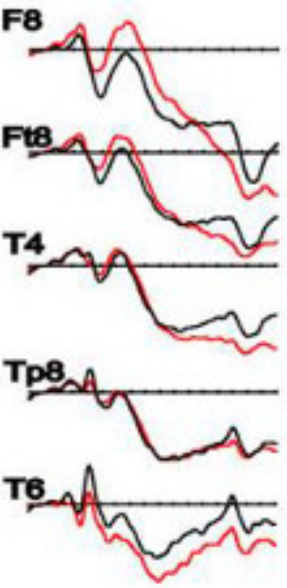

Figure 4. Effect of Schizotypy. This figure depicts the grand average ERPs in the placebo condition. Red lines correspond to participants with high scores on the schizotypal personality questionnaire. Black lines correspond to participants with low schizotypy as determined by the questionnaire.
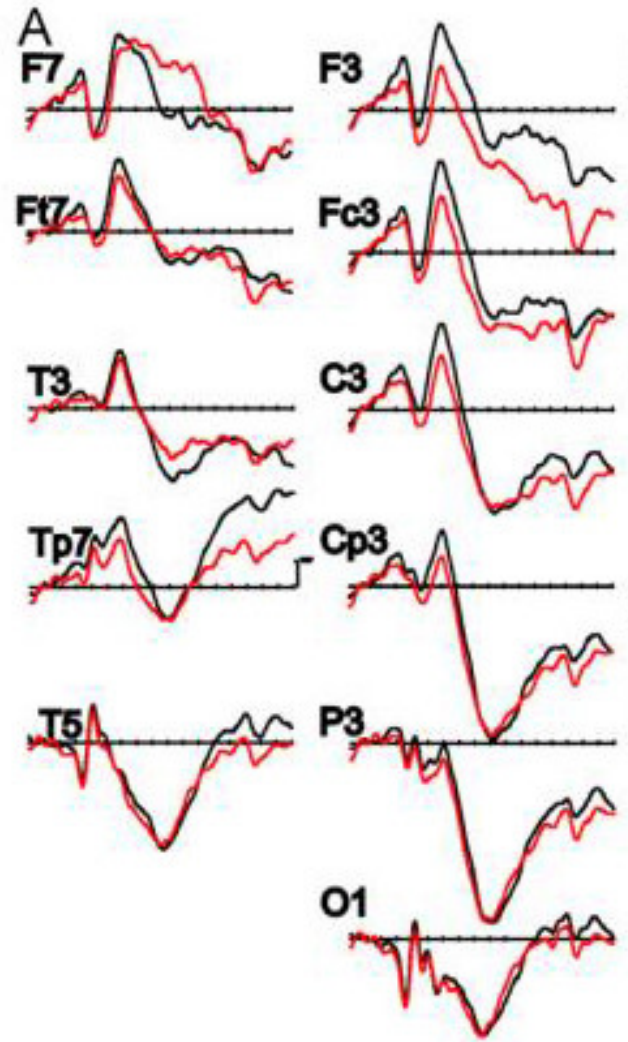
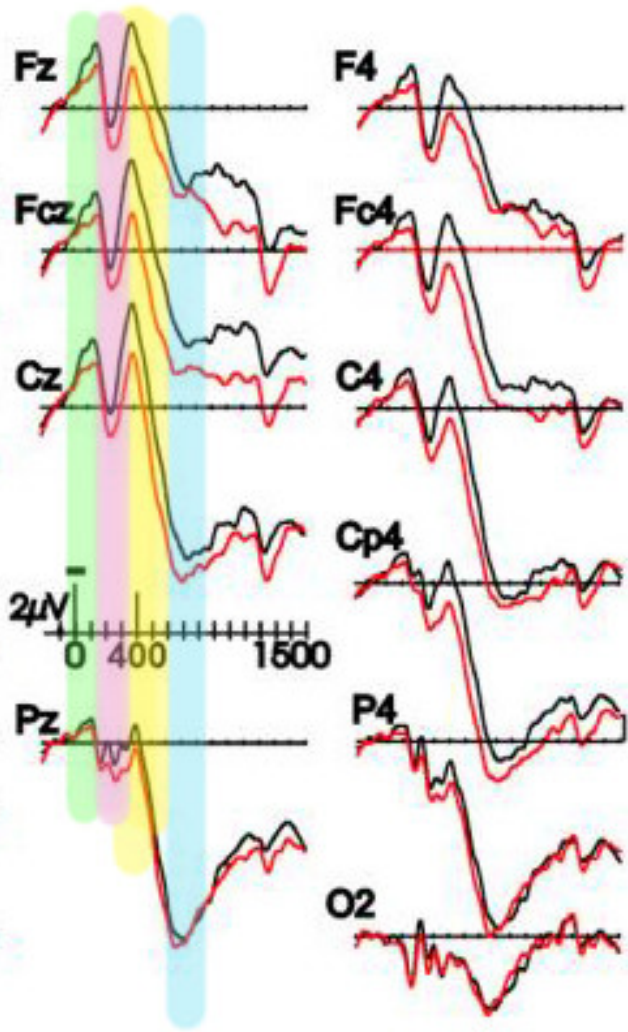

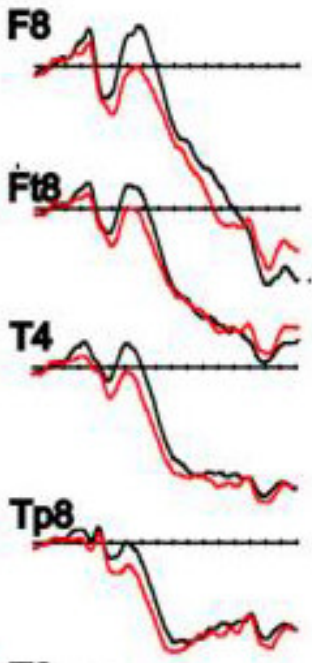

T6

Figure 5. Effects of medication in high schizotypy individuals. This figure depicts the grand average ERPs, where red corresponds to medication (Olanzapine), and black corresponds to placebo. Please click here to view a larger version of this figure.

Animated Figure 1. This figure can be used as a guide for processing the data and obtaining ERPs.

\section{Discussion}

In our investigation of the effects of antipsychotic medication on the N400, we found that in the placebo condition, anterior N400s were larger in individuals with high schizotypy than in the subjects with low schizotypy. In the medication condition however, fronto-central N400s were smaller, 
but only in the individuals with high schizotypy. Thus, the medication was seen to decrease these large fronto-central N400s in high schizotypal individuals, which confirms previous research illustrating smaller anterior N400s in schizophrenic patients treated with antipsychotic medication, relative to patients receiving a placebo ${ }^{11,12}$. In individuals with low schizotypy, there was no effect of medication. These findings were obtained with the proper implementation of the protocol, and speak to the importance of collecting clear and precise data.

To ensure accurate and valid results, there are a few critical points to keep in mind. To get a good signal, it is crucial that the cap is snugly fit and placed so that the sagittal electrodes are exactly in the middle and that impedance is adjusted. A cap that is too large can reduce the recording quality, as it will be much harder to reduce impedances ${ }^{13}$. It is also important that the participants understand that they must avoid excessive movement, blinking, or flexing their face and jaw muscles, as these actions will introduce changes in the EEG traces and potentially make data interpretation very difficult ${ }^{14}$. After the experiment, the equipment must be properly cleaned to ensure that the electrodes are not blocked with gel residue, which could affect future signal collection.

If there are problems in the signal, such as noise or flat lines, check to make sure that both the ground and reference electrodes are properly connected. Reducing the impedance of these electrodes can reduce noise, so if there are problems in the connectivity, reapply the gel and rescratch the scalp underneath the electrodes. If there are myograms, allow the subject to relax before proceeding. In designing the experiment, it is relevant for ERP recording that the experimental design accounts for timing aspects, such as the duration of stimulus presentation and brief time periods that allow the participant to blink.

Before beginning an EEG experiment, it is important to understand the associated limitations. The relatively poor spatial resolution may be something to consider, as well as the difficulty of using EEG to elucidate subcortical activity. The noise introduced by blinks and muscle activity is also disadvantageous, and the neural signals obtained are smudged as they pass through the cerebrospinal fluid, meninges, and skull. These limitations may be addressed with alternative neuroimaging methods independently- such as fMRI, NIRS - or by combining EEG with these alternate approaches. However, as previously mentioned, these approaches also carry their own limitations. The requisite contrast conditions and the lack of baseline and polarity in fMRI are notable examples. With respect to alternative brain imaging techniques, ERP recording has many advantages- most particularly its remarkable temporal resolution, which is at the level of milliseconds. It is also non-invasive and minimally uncomfortable for the participant, and is not associated with any significant risks or dangers. ERP recording is economically sound, especially when compared to other neuroimaging techniques such as functional magnetic resonance imaging (fMRI) and positron emission tomography $(\mathrm{PET})^{15}$. It offers a look into the neural events that occur in cognitive, sensory, and motor processes, and in the future, could be applied to explore preconscious processes.

\section{Disclosures}

The authors have nothing to disclose.

\section{Acknowledgements}

The authors would like to acknowledge the Brain and Behavior Research Foundation for their generous support.

\section{References}

1. Debruille, J. B., Rodier, M., Prevost, M., Lionnet, C., \& Molavi, S. Effects of a small dose of olanzapine on healthy subjects according to their schizotypy: an ERP study using a semantic categorization and an oddball task. Europeam Neuropsychopharmacology. 23 (5), $339-350$, doi: 10.1016/j.euroneuro.2012.06.005 (2013).

2. Bressler, S.L. Event-related potentials. The Handbook of Brain Theory and Neural Networks., Arbib, M.A., ed., MIT Press, Cambridge, MA, 412-415 (2002).

3. Peterson, N. N., Schroeder, C. E., \& Arezzo, J. C. Neural generators of early cortical somatosensory evoked potentials in the awake monkey. Electroencephalography and Clinical Neurophysiology. 96 (3), 248-260, doi: 10.1016/0168-5597(95)00006-E (1995).

4. Blackwood, D. H., \& Muir, W. J. Cognitive brain potentials and their application. The British Journal of Psychiatry Supplement. 9, 96-10 (1990).

5. Debruille, J. B. The N400 potential could index a semantic inhibition. Brain Research Reviews. 56 (2), 472-477, doi: 10.1016/ j.brainresrev.2007.10.001 (2007).

6. Kutas, M., \& Hillyard, S. A.Event-Related Brain Potentials (ERPs) Elicited by Novel Stimuli during Sentence Processing. Annals of the New York Academy of Sciences. 425 (1), 236-241, doi: 10.1111/j.1749-6632.1984.tb23540.x (1984).

7. Holcomb, P.J., Kounios, J., Anderson, J.E, West, W.C Dual-coding, context-availability, and concreteness effects in sentence comprehension: an electrophysiological investigation. Journal of Experimental Psychology: Learning, Memory, and Cognition. 25, 721-742, doi: 10.1037/0278-7393.25.3.721 (1999).

8. Renoult, L., Brodeur, M.B., Debruille, J.B. Semantic processing of highly repeated concepts presented in single-word trials: electrophysiological and behavioral correlates. Biological Psychology. 84, 206-220, doi: 10.1016/j.biopsycho.2010.01.014 (2010).

9. Prevost, M. et al., Schizotypal traits and N400 in healthy subjects. Psychophysiology. 47, 1047-1056, doi: 10.1111/j.1469-8986.2010.01016.x (2010).

10. Sur, S., \& Sinha, V. K. Event-related potential: An overview. Industrial Psychiatry Journal. 18 (1), 70-73, doi: 10.4103/0972-6748.57865 (2009).

11. Condray, R., Siegle, G.J., Cohen, J.D., van Kammen, D.P., Steinhauer, S.R., Automatic activation of the semantic network in schizophrenia: evidence from event-related brain potentials. Biological Psychiatry. 54, 1134-1148, doi: 10.1016/S0006-3223(03)00699-1 (2003).

12. Condray, R., Steinhauer, S.R., Cohen, J.D., van Kammen, D.P., Kasparek, A. Modulation of language processing in schizophrenia: effects of context and haloperidol on the event-related potential. Biological Psychiatry. 45, 1336-1355, doi: 10.1016/S0006-3223(98)00317-5 (1999). 
13. Light, G. A., et al. Electroencephalography (EEG) and event-related potentials (ERPs) with human participants. Current Protocols in Neuroscience. 21-24, doi: 10.1002/0471142301.ns0625s52 (2010).

14. Luck, S. J. Basic Principles of ERP Recording. An Introduction to the Event-Related Potential Technique. MIT Press, Cambridge, Mass (2005).

15. Luck, S. J. An Introduction to Event-Related Potentials and Their Neural Origins. An Introduction to the Event-Related Potential Technique. MIT Press, Cambridge, Mass (2005). 